\title{
Evaluation of Ischemia Following Clipping of Anterior Circulation Aneurysms with Respect to Temporary Clipping Using Diffusion-Weighted Magnetic Resonance Imaging: A Prospective Study
}

\author{
Pankaj Kumar ${ }^{1}$ Shaam Bodeliwala ${ }^{2}$ Rajender Aher \\ Daljit Singh² Pragati Ganjoo²
}

Anita Jagetia ${ }^{2}$ Arvind Kumar Srivastava²

\begin{abstract}
Address for correspondence Shaam Bodeliwala, MCh (Neurosurgery), Department of Neurosurgery, G.B. Pant Institute of Postgraduate Education and Research (GIPMER), New Delhi 110002, India (e-mail: shaam.86@gmail.com).
\end{abstract}

\begin{abstract}
Keywords

- aneurysm

- cerebral infarction

- intraoperative

hemorrhage

Temporary vessel occlusion enables a surgeon dissect aneurysm and clip with a lower risk of intraoperative hemorrhage with the associated risk of ischemia. There are studies on permissible time of occlusion of the parent artery using temporary clip; however, the actual incidence of silent ischemic events in patients with aneurysms treated with microsurgical clipping is not well documented. We are trying to look for the association between temporary clipping and incidence of ischemia through this study. The study concluded the statistically significant association between the maximum time of single clip application and ischemia. Intermittent multiple temporary clippings can prevent ischemia instead of a single clipping of longer duration.
\end{abstract}

\section{Introduction}

Temporary vessel occlusion enables a surgeon dissect aneurysm and clip with a lower risk of intraoperative hemorrhage with the associated risk of ischemia. There are studies on permissible time of occlusion of parent artery using temporary clip; however, the actual incidence of silent ischemic events in patients with aneurysms treated with microsurgical clipping is not well documented. The ischemia may manifest or may be silent. Since silent ischemia does not manifest clinically, it can be picked by various means such as positron emission tomography scans, single photon emission computed tomography scan, or various sequences of magnetic resonance imaging (MRI). This study is done to analyze the risk of symptomatic and silent ischemia in association with temporary clipping used during the microsurgical clipping of intracranial aneurysms using diffusion-weighted magnetic resonance imaging (DWMRI) and to evaluate the permissible time which can cause even silent ischemia. It was used in our study due to the availability, feasibility, and cost effectiveness of DWMRI.

\section{Materials and Methods}

This study evaluates patients in World Federation of Neurological Surgeons (WFNS) grades I to III, operated for anterior circulation cerebral aneurysms over 3 years by craniotomy and clipping of aneurysm by a single surgeon. Anesthesia given to them was as per the standard protocol for aneurysm clipping. During surgery, the number of temporary clips applied and the time period for which they were applied were recorded. Other causes for possible ischemic events had been ruled out. DWMRI brain was done prior to surgery and within 24 hours of surgery in all study patients and ischemia signs noted by an experienced neuroradiologist and correlated with patient's post clipping neurological status and categorized as asymptomatic and or symptomatic ischemia.
DOI https://doi.org/

10.1055/s-0039-3402625

ISSN 2277-954X.
License terms

()(1) $\Theta \circledast$ 


\section{Statistical Analysis}

Baseline demographics of the study population were analyzed using descriptive statistical parameters. For comparisons of categorical variables, Fischer's exact or chi-square tests are used. For comparisons of continuous variables, the rank-sum or equivalent Mann-Whitney $U$ test is used. For all analyses, $p<0.05$ was considered statistically significant. All computations were performed using the commercially available software programs and statistician's aid.

\section{Results}

Out of 21 patients, 18 were in the WFNS grade I, one was in grade II, and two were in grade III. Most common age group was 31 to 40 years consisting of eight patients in grade I, followed by 41 to 50 years consisting of five patients in grade I, one in grade II, and one in grade III. The mean age of presentation was 39.8 years. Out of a total of 21 patients, $12(57.1 \%)$ were males and $9(42.8 \%)$ were females with male to female ratio of $4: 3$. Headache was the commonest presenting symptom in all patients followed by vomiting and loss of consciousness. Hemiparesis was reported in only two patients.

Anterior communicating artery aneurysms constituted 11 (52.3\%) patients, 7 (33.3\%) patients had middle cerebral artery (MCA) aneurysms, 2 (9.5\%) patients had supraclinoid internal carotid artery (ICA) aneurysms, and only one (4.7\%) patient was harboring aneurysms at the junction of ICA-posterior communicating artery.

Out of 21 patients, ischemic changes were noted in five (23.8\%) patients on immediate postoperative DWMRI study. Subgroup analysis as per the size of the aneurysm revealed only one (33.3\%) case of asymptomatic ischemia and no case developed symptomatic ischemia clinically in small $(<7 \mathrm{~mm})$ size aneurysm group. Out of 14 cases of medium (7-14 mm) size group, one (7.1\%) patient developed symptomatic infarct and two (14.2\%) patients developed asymptomatic infarcts. One patient (33.3\%) out of three cases of large-size aneurysms (15-24 mm) size developed symptomatic infarct (- Table 1).

In $17(81 \%)$ cases, temporary clip was applied, out of which two (11.7\%) and three (17.6\%) patients developed symptomatic and asymptomatic ischemia, respectively (-Figs. 1 and $\mathbf{2}$ ). No ischemic changes were noted in four patients in whom temporary clip was not used.

The average total mean time of temporary clip application, leading to symptomatic as well as DWMR-proved ischemic cases, was 300 seconds. In asymptomatic but
DWMR-proved ischemic cases, the average total mean time was 123.33 seconds, however, it was 127.75 seconds in patients without any ischemic changes on DW study (-Fig. 3).

Single maximum mean time of the temporary clip used was 145 seconds in both symptomatic and asymptomatic post clipping ischemic cases. In patients without any post clipping ischemic changes, the maximum mean single time use of temporary clip was only 81.93 seconds.

Based on the average number of temporary clips applied, i.e., number of vessels in which temporary clips were applied, was 3.5 in cases of postoperative symptomatic ischemia, whereas average 2.33 and 1.62 clips were applied in asymptomatic ischemic cases and in patient without any post clipping ischemia on DWMRI respectively (-Fig. 1).

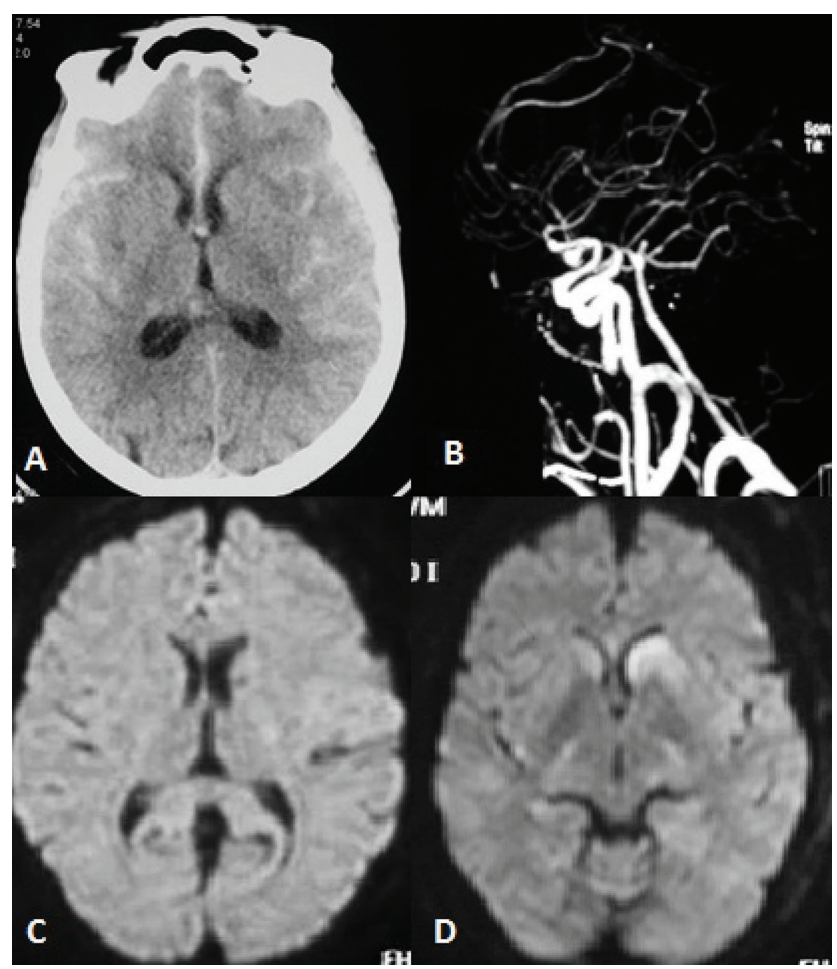

Fig. 1 Radiological images of the patient with asymptomatic infarction. (A) NCCT head on admission showed subarachnoid hemorrhage; (B) Preoperative angiography revealed anterior communicating artery aneurysm; (C) Preoperative DWMRI showed no infarct; (D) Immediate postoperative DWMRI showed a small asymptomatic infarct in the left basal ganglion region. DWMRI, diffusion-weighted magnetic resonance imaging; NCCT, noncontrast computed tomography.

Table 1 Aneurysm size vs. postoperative ischemia

\begin{tabular}{|l|l|l|l|l|}
\hline Size of aneurysm & $\begin{array}{l}\text { Symptomatic } \\
\text { ischemic cases }\end{array}$ & $\begin{array}{l}\text { Asymptomatic ischemic } \\
\text { cases }\end{array}$ & Nonischemic cases & Total \\
\hline Small $(<7 \mathrm{~mm})$ & 0 & $1(33.3 \%)$ & $2(66.6 \%)$ & 3 \\
\hline Medium $(7-14 \mathrm{~mm})$ & $1(7.1 \%)$ & $2(14.2 \%)$ & $11(78.5 \%)$ & 14 \\
\hline Large $(15-24 \mathrm{~mm})$ & $1(33.3 \%)$ & 0 & $2(66.6 \%)$ & 3 \\
\hline Giant $(\geq 25 \mathrm{~mm})$ & 0 & 0 & 1 & 1 \\
\hline Total & 2 & 3 & 16 & 21 \\
\hline
\end{tabular}

Note: The table shows occurrence of ischemia in different groups according to the size of aneurysms. 


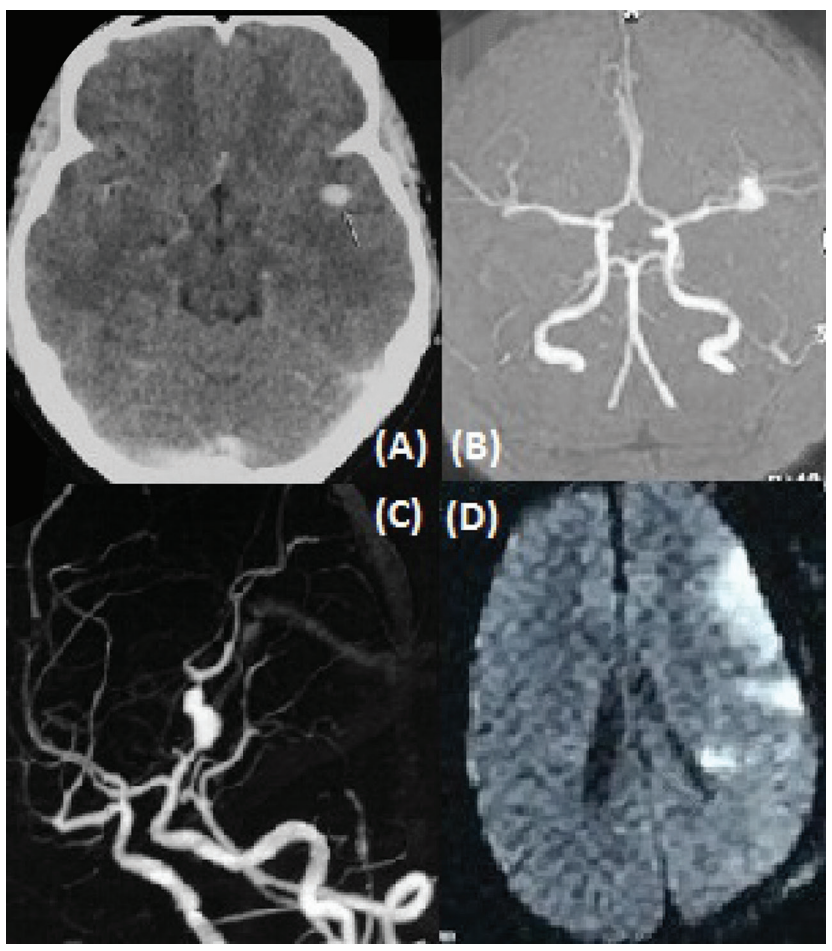

Fig. 2 Radiological images of the patient with symptomatic infarction. (A) NCCT head on admission showed subarachnoid hemorrhage; $(\mathbf{B}, \mathbf{C})$ CT angiography brain suggested left MCA bifurcation aneurysm; (D) The patient was operated for the same and developed symptomatic left side MCA territory infarct as shown in immediate postoperative DWMRI. CT, computed tomography; DWMRI, diffusion-weighted magnetic resonance imaging; MCA, middle cerebral artery; NCCT, noncontrast computed tomography.
Glasgow outcome scale at 1 month from discharge was score 4 in post clipping symptomatic infarct cases. It was 5 in both asymptomatic infarct cases and in patients without ischemia. Finally, in this study, out of 21 patients, $9.52 \%$ patients developed symptomatic ischemic changes, $14.2 \%$ patients developed asymptomatic ischemic changes on DWMRI.

\section{Discussion}

Patients in whom cerebral infarction develops after aneurysmal subarachnoid hemorrhage ( $\mathrm{SAH}$ ) are less likely to make a good recovery and more likely to die or survive with deficit than those without infarction. ${ }^{1}$ In the present study also Glasgow outcome scale at the time of discharge was statistically significant in post clipping ischemic group compared with the nonischemic group.

Juvela et $\mathrm{al}^{2}$ have reported incidence of ischemia to be $65 \%$ on postoperative CT scan. Krayenbühl et al $^{3}$ reported the incidence of symptomatic and asymptomatic ischemia to be 2 and $9.5 \%$, respectively. Ferch et $\mathrm{al}^{4}$ also found $17 \%$ of symptomatic and $26 \%$ of radiologically evident ischemic changes after the use of temporary clip. In this study, symptomatic and asymptomatic ischemic changes happened in 9.52 and $14.20 \%$ patients, respectively as evidenced by DWMRI sequence. Overall, $23.72 \%$ of our study population developed ischemic changes where temporary clipping was used during surgeries, which is consistent with the study. Cronqvist et $\mathrm{al}^{5}$ reported over all silent and symptomatic ischemia rate of $23 \%$ using diffusion and perfusion MRI in patients with ruptured and unruptured

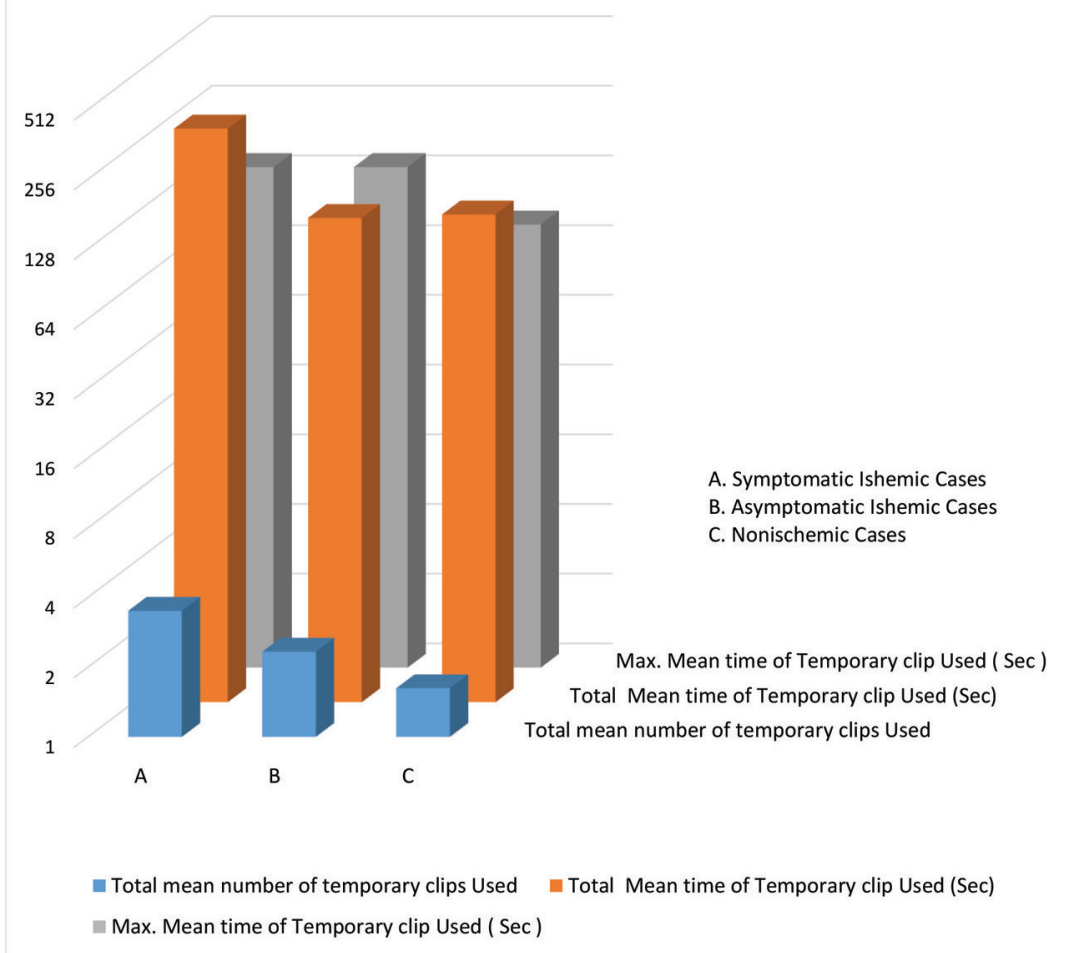

Fig. 3 Number, total mean time, and maximum mean time vs. postoperative ischemia. 
intracranial aneurysms treated by endovascular coiling which is comparable to our study result of $23.8 \%$ in post clipping group in spite of taking advantage of full anticoagulation in endovascular treatment.

Median age of patients in post clipping ischemic group was 50 and 40 years in nonischemic group, but univariate analysis suggested no significant statistical relation of age with post clipping ischemic changes. There is no statistically significant difference between sex, size, and location of aneurysm between post clipping ischemic group and no ischemic group as is previously reported. ${ }^{3}$

It has previously been suggested that the use of temporary clipping during microsurgery of aneurysms leads to ischemic events. ${ }^{4,5}$ The duration of temporary clipping was found to correlate with the increased occurrence of ischemic events. ${ }^{3,6}$ Selvapandian et $\mathrm{al}^{7}$ found no statistically significant correlation between occurrence of complications and total duration of temporary clipping or number of temporary clippings. Whereas this study shows the multiple use of temporary clip does not cause postoperative ischemia but single application of the clip for a longer period (single maximum time of temporary clip application, $p$-value $=0.01248)$ may influence the occurrence of both silent and symptomatic ischemia (-Table 2). According to our study, the acceptable duration of one-time temporary clip may be less than 145 seconds that can avoid symptomatic ischemic complication to happen, however, the duration should be kept minimum possible to less than 90 seconds to avoid any early DWMRI ischemic changes as it might result in symptomatic ischemia in particular case. The total time of temporary clip application was much less in our study as of $585.4 \pm 858.4$ seconds in contrast to other studies reported. ${ }^{1}$ Although Ogilvy et $\mathrm{al}^{8}$ suggested temporary clip application up to 20 minutes, in this study the critical time period of temporary clip application is 5 minutes, that is, 300 seconds. However, to prove, this would require further intraoperative study such as Doppler and intraoperative video angiographies to assess changes in blood flow happening at the time of temporary clipping (-Table 2).

Table 2 Univariate analysis showing association of ischemia with various factors

\begin{tabular}{|c|c|c|c|}
\hline Factors & Ischemic group $(n=5)$ & Nonischemic group $(n=16)$ & $p$-Value \\
\hline Age & $50 \pm 18$ & $40 \pm 9$ & 0.4949 \\
\hline Male/Female (\%) & $1(20 \%) / 4(80 \%)$ & $11(68.7 \%) / 5(31.3 \%)$ & 0.1194 \\
\hline \multicolumn{3}{|l|}{ Location of aneurysm } & \multirow[t]{5}{*}{0.1474} \\
\hline ACOM artery & 1 & 10 & \\
\hline MCA & 2 & 5 & \\
\hline ICA bifurcation & 1 & 1 & \\
\hline ICA-PCOM & 1 & 0 & \\
\hline \multicolumn{3}{|l|}{ Size } & \multirow[t]{5}{*}{0.6539} \\
\hline Small & 1 & 2 & \\
\hline Medium & 3 & 11 & \\
\hline Large & 1 & 2 & \\
\hline Giant & 0 & 1 & \\
\hline \multicolumn{3}{|l|}{ Temporary clip used } & \multirow[t]{3}{*}{0.5322} \\
\hline Yes & 5 & 12 & \\
\hline No & 0 & 4 & \\
\hline Number of times temporary clip used & $30 \pm 0.84$ & $2 \pm 1.41$ & 0.0752 \\
\hline $\begin{array}{l}\text { Single maximum time of temporary clip } \\
\text { application (sec) }\end{array}$ & 145 & 81.93 & $0.01248(<0.05)$ \\
\hline $\begin{array}{l}\text { Total time of temporary clip application } \\
(\mathrm{sec})\end{array}$ & 254 & 127.75 & $0.00815(<0.05)$ \\
\hline Mean GOS at 1 month & 4.6 & 5 & 0.2079 \\
\hline \multicolumn{3}{|l|}{ WFNS grade } & \multirow[t]{4}{*}{0.1074} \\
\hline 1 & 3 & 15 & \\
\hline II & 1 & 0 & \\
\hline III & 1 & 1 & \\
\hline
\end{tabular}

Abbreviations: ACOM, anterior communicating; GOS, Glasgow outcome scale; ICA, internal carotid artery; MCA, middle cerebral artery; PCOM, posterior communicating artery; WFNS, World Federation of Neurological Surgeons.

Note: For categorical variables-Fischer's exact or chi-square tests were used and for continuous variables, the rank-sum or equivalent Mann-Whitney $U$ test was used. Significant $p$-value $<0.05$ is mentioned in bold letters. 


\section{Conclusion}

The most significant factors associated with the occurrence of post clipping cerebral ischemic changes are maximum time of single clip application and total time of temporary clips application. The incidence of ischemic changes occurring following temporary clip placement is more or less same as reported in endovascular treatment despite the fact that latter procedure is taking the advantage of anticoagulation at the time of intervention.

\section{Funding}

None.

\section{Conflict of Interest}

None.

\section{References}

1 Adams HP Jr, Kassell NF, Torner JC, Haley EC Jr. Predicting cerebral ischemia after aneurysmal subarachnoid hemorrhage: influences of clinical condition, CT results, and antifibrinolytic therapy. A report of the Cooperative Aneurysm Study. Neurology 1987;37(10):1586-1591

2 Juvela S, Siironen J, Kuhmonen J. Hyperglycemia, excess weight, and history of hypertension as risk factors for poor outcome and cerebral infarction after aneurysmal subarachnoid hemorrhage. J Neurosurg 2005;102(6):998-1003

3 Krayenbühl N, Erdem E, Oinas M, Krisht AF. Symptomatic and silent ischemia associated with microsurgical clipping of intracranial aneurysms: evaluation with diffusion-weighted MRI. Stroke 2009;40:129-133

4 Ferch R, Pasqualin A, Pinna G, Chioffi F, Bricolo A. Temporary arterial occlusion in the repair of ruptured intracranial aneurysms: an analysis of risk factors for stroke. J Neurosurg 2002;97(4):836-842

5 Cronqvist M, Wirestam R, Ramgren B, et al. Diffusion and perfusion MRI in patients with ruptured and unruptured intracranial aneurysms treated by endovascular coiling: complications, procedural results, MR findings and clinical outcome. Neuroradiology 2005;47(11):855-873

6 Samson D, Batjer HH, Bowman G, et al. A clinical study of the parameters and effects of temporary arterial occlusion in the management of intracranial aneurysms. Neurosurgery 1994;34(1):22-28, discussion 28-29

7 Selvapandian S, Sudarsan PS, Shaji PG, Chandy MJ. Elective intermittent temporary clipping in aneurysm surgery: a practical protocol. Indian J Neurosurg 2015;04:8-14

8 Ogilvy CS, Carter BS, Kaplan S, Rich C, Crowell RM. Temporary vessel occlusion for aneurysm surgery: risk factors for stroke in patients protected by induced hypothermia and hypertension and intravenous mannitol administration. J Neurosurg 1996;84(5):785-791 